\title{
Onset and evolution of discontinuously segmented chip flow in ultra-high-speed cutting Ti-6Al-4V
}

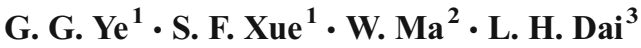

Received: 27 October 2015 / Accepted: 25 April 2016 /Published online: 6 May 2016

(C) Springer-Verlag London 2016

\begin{abstract}
Increasing the cutting speed to an ultra-high level usually gives rise to a chip flow transition from continuously serrated to discontinuously segmented, which is one of the most fundamental and challenging problems in metal cutting. In this work, we experimentally performed the ultra-high-speed cutting on Ti-6Al$4 \mathrm{~V}$ with a maximum cutting speed of $210 \mathrm{~m} / \mathrm{s}$, focusing on the physical phenomena accompanying the discontinuously segmented chip flow. It reveals that the discontinuously segmented chip flow can be attributed to the shear fracture induced by the fully matured shear banding, and there exists a ductile-brittle transition of the shear fracture as the cutting speed increases to an ultra-high level. In addition, the critical condition for the onset of segmented chip flow is presented using the momentum diffusion-based shear band evolution model, which gives good prediction for the chip segmentation.
\end{abstract}

Keywords High-speed machining · Shear banding ·

Segmented chip · Discontinuous chip · Momentum diffusion

L. H. Dai

lhdai@lnm.imech.ac.cn

1 Department of Engineering Mechanics, College of Pipeline and Civil Engineering, China University of Petroleum, Shandong 266580, China

2 Key Laboratory of Environmental Mechanics, Institute of Mechanics, Chinese Academy of Sciences, Beijing 100190, China

3 State Key Laboratory of Nonlinear Mechanics, Institute of Mechanics, Chinese Academy of Sciences, Beijing 100190, China

\section{NomenclatureGreek alphabet}

$\alpha \quad$ Thermal softening coefficient

$\gamma \quad$ Shear strain

$\gamma_{0} \quad$ Shear strain rate

$\gamma_{\text {ref }} \quad$ Reference strain rate in J-C law

$\eta \quad$ Strain rate-hardening coefficient

$\theta \quad$ Temperature rise

$\lambda$ Thermal diffusivity

$\mu \quad$ Tool-chip friction coefficient

$\xi \quad$ The width of rigid region

$\rho \quad$ Work material density

$\tau \quad$ Shear band stress

$\tau_{y} \quad$ The initial yield shear stress

$\tau_{A} \quad$ Initial yield stress in J-C law

$\tau_{B} \quad$ Hardening modulus in J-C law

$\varphi \quad$ Shear angle

$\psi \quad$ Shear band slip distance

$\omega \quad$ Tool rake angle

$\Gamma \quad$ Energy dissipated in shear banding

\section{English alphabet}

a Shear band thickness

$b \quad$ Uncut chip thickness

$c \quad$ Strain gradient coefficient

$C$ Strain rate coefficient in J-C law

$C_{p} \quad$ Thermal capacity

$h \quad$ PSZ thickness

$t \quad$ Shear band evolution time

$V \quad$ Cutting speed

$V_{s} \quad$ Tool velocity along shear direction

$V_{f} \quad$ Material convection velocity

$X \quad$ Shear band evolution degree

$W_{\text {input }} \quad$ Energy inputted into PSZ

\section{Subscript}

$c$ Critical condition at which the shear band is fully matured

$u$ The ultimate evolution condition for the shear band in serrated chip flow 


\section{Parameter combination}

$\bar{a}$

$$
a\left(9 \rho^{3} c^{2} \lambda^{3} / \tau_{y}^{3} \alpha^{2} \gamma_{0}\right)^{-1 / 4}
$$

$R$

$$
2 \eta \sqrt{\gamma_{0} / \rho \lambda \tau_{y}}
$$

$S$

$$
c\left(\gamma_{0} / \tau_{y} \rho \lambda^{3}\right)^{1 / 2}
$$

$\bar{\Gamma}$

$$
\Gamma \alpha\left(9 \rho^{3} c^{2} \lambda^{3} / \tau_{y}^{3} \alpha^{2} \gamma_{0}\right)^{-1 / 4} /(2 \rho c)
$$

$M \quad V_{f} a_{0} / 2 \lambda$

\section{Introduction}

The growing demand for enhancing production efficiency and product quality has led to the rapid development of high-speed cutting or machining technology [1-3]. In spite of extensive studies, several fundamental aspects of the high-speed cutting process are poorly understood. One of these aspects is the onset of discontinuously segmented chip flow.

There usually exist two distinct transitions of the chip flow pattern as the cutting speed increases from low to very high. The chip flow is usually continuously smooth at low cutting speed. Increasing the cutting speed leads to a breakdown of the steady chip flow and results in a serrated chip flow pattern. The other transition, from continuously serrated to discontinuously segmented chip flow, usually occurs at very high cutting speeds. These two transitions of chip flow pattern are two of the most fundamental and challenging problems in metal cutting.

The formation of continuously serrated chip flow has been extensively studied [4-7]. It is found that the emergence of serrated chip flow is related to the cracking initiated at the free surface $[6,8]$ or the shear banding occurred in the primary shear zone (PSZ) [9-15]. A number of researchers studied the occurrence condition of shear banding in high-speed machining, and several classical models have been developed to derive the condition under which the continuously smooth chip flow becomes unstable [16-23].

With regard to discontinuously segmented chip flow, it usually forms at very high cutting speeds. It is favored for machined components because continuously smooth and serrated chips get tangled and are not appropriate for automated processes. The discontinuous segmentation is however believed to be a critical aspect due to periodic variations in the cutting forces that increase tool wear rate and degradation of the machined surface finish. The causes and effects of chip segmentation have received important attention in the aim of selecting the optimal cutting conditions to improve the production and increase both the tool life and surface quality. Hitherto, however, the formation mechanism of discontinuously segmented chip flow, which usually occurs at very high cutting speed, has not been well understood. Only a few researches have dealt with the formation mechanism of discontinuous chip. The puzzle of why serrated chip flow gives way to discontinuously segmented chip flow as the cutting speed increases to a extremely high level still remains elusive.

Sowerby and Chandrasekaran [24] presented a method to predict the chip segmentation using a critical damage factor. Later, Marusich and Ortiz [25] estimated the crack propagation in serrated chip using the dynamic fracture factor together with the maximum loop stress criterion. They found that discontinuous chip flow usually occurs at negative rake angle. Based on the theory of strain energy density, Lin and his coworkers [26, 27] developed an elastic-plastic finite element model (FEM) to simulate the discontinuous chip formation. Also, using the FEM method, Guo and Yen [28] simulated the chip crack initiation and propagation in discontinuous chip. They stated that the discontinuous chip is due to the internal crack initiation and extension in front of the tool and meeting with the surface crack, and the adiabatic shearing was found to play an important role in discontinuous chip formation. More recently, Liu and $\mathrm{Su}$ [29] studied the correlation between the chip morphology and the dynamic mechanical properties of work material; they attributed the discontinuous chip flow to the brittle fracture occurred in high-speed cutting. Using a specific ballistic setup together with a high-speed imaging system, Sutter and List [30] investigated the transition of serrated chip to discontinuously segmented chip. They stated that the chip segmentation is governed by the phenomena of shear band propagation and crack formation. Gu et al. [31] carried out the high-speed cutting experiments on AISI 1045 steel with a maximum speed of $23 \mathrm{~m} / \mathrm{s}$. The adiabatic shear localized fracture is claimed to be the key reason for chip segmentation, and a theoretical model is further proposed to achieve the fracture criterion. Recently, Cui et al. [32] showed in their work that the high temperature in the area between the adjacent sawteeth has a great effect on the formation of discontinuous chip.

These pioneering works provide important clues to study the discontinuously segmented chip flow. However, the range of very high cutting speeds when machining metal alloys is still largely unexploited. The formation mechanism and, especially, the onset condition for the discontinuously segmented chip flow are so far unclear. It should be pointed out that the discontinuously segmented chip formation involves highly nonlinear thermo-viscoplastic flow and fracture of material. And, the coupled effects of momentum diffusion and thermal diffusion can become extremely complex at very high speeds. All of these factors make the problem much more complex, and the emergence of discontinuously segmented chip flow still remains one of the least understood manufacturing phenomena. In the present work, orthogonal cutting experiments 
were carried out over a very wide range of cutting speeds to illustrate the reason for the emergence of discontinuously segmented chip flow. Furthermore, we developed the momentum diffusion-based shear band evolution model, which was first established in our previous work, to predict the onset of discontinuous chip flow.

\section{Experimental}

The titanium alloy Ti-6Al-4V, which is widely employed in the aerospace industries, was chosen as workpiece. The chemical compositions and heat treatments for the Ti-6Al-4V alloy are given in Table 1.

To reproduce high-speed cutting process, an original experimental setup was developed [33] and schematically presented in Fig. 1, and the actual experimental setup is presented in Fig. 2. The cutting tool is propelled by a light-gas gun in a launch tube, and two symmetrical workpieces are fixed at the exit of the tube. Orthogonal cutting occurs when the tool impacts the workpieces. To achieve ultra-high cutting speeds, we reduced the weight of the projectile and elevated the gas pressure, and a maximum cutting speed of $210 \mathrm{~m} / \mathrm{s}$ was achieved.

In addition, to investigate the evolution of the chip flow from low to high cutting speeds and to capture the two significant transitions of the chip flow pattern, the low-speed cutting experiments were also performed, using a computercontrolled lathe.

In this way, orthogonal cutting experiments were performed on the Ti-6Al-4V alloy over a very wide range of cutting speeds, from 0.05 to $210 \mathrm{~m} / \mathrm{s}$. The uncut chip thickness was set to be $100 \mu \mathrm{m}$ and the tool rake angle to be $0^{\circ}$. Uncoated P10 carbide tools with a tool edge radius of $0.01 \mathrm{~mm}$ were applied. To minimize the influence of cutting tool wear on experimental results, the cutting length was limited, and the cutting insert was replaced after each cutting.

Chips generated were collected and observed by a highresolution scanning electron microscopy.

\section{Results and discussion}

\subsection{The evolution of chip flow pattern}

The evolution of chip flow pattern with cutting speed for Ti$6 \mathrm{Al}-4 \mathrm{~V}$ is shown in Fig. 3.
Here, to show clearly the whole evolution process of the chip flow pattern from low to ultra-high cutting speeds and, thus, to reveal the underlying mechanism of the initiation of discontinuously segmented chip flow, the morphology images of the chips obtained at low cutting speeds are shown together with that obtained at high cutting speeds, although the lowseed cutting experimental results for Ti-6Al-4V have been widely reported [34-36].

At very low cutting speeds, chips undergo a relatively homogenous shear deformation, giving rise to a continuously smooth chip flow pattern (see Fig. 3a). At higher cutting speeds, the thermoplastic instability-induced shear banding emerges because the smooth chip flow is not sufficient to dissipate the energy through homogeneous plastic flow (see Fig. 3c, g). This results in a serrated or sawtooth-like chip flow pattern, which is still continuous.

As increasing the cutting speed to a higher level, the shear band between the "sawteeth" evolves much more sufficiently, leading to a higher serration degree. At the cutting speed of $68.9 \mathrm{~m} / \mathrm{s}$, the chip is highly serrated. In this case, a few shear bands developed to a significant high level, and cracking occurs near the roots of these shear bands. Consequently, the corresponding sawteeth separate with their adjacent ones along these shear bands, although most sawteeth are still continuously connected (see Fig. 3e, h).

When the cutting speed is elevated up to $86.5 \mathrm{~m} / \mathrm{s}$, all the sawteeth separate with each other, and the chip turns out to be discontinuously segmented, as shown in Fig. 4. Each isolated chip is a piece of "sawtooth."

\subsection{The microstructure evolution before segmentation}

The evolution of the microstructure of the chip free surface with cutting speed (before segmentation) is shown in Fig. 5.

It can be noted from Fig. 5a-d that higher cutting speed results in more remarkable shear front surfaces, implying that increasing the cutting speed promotes the evolution of the shear band.

As the cutting speed increases to $68.9 \mathrm{~m} / \mathrm{s}$, the shear front surfaces are especially obvious. Most areas of the shear front surfaces show severe elongated dimple structures, which is a most significant feature of the shear localization fracture [37]. In this case, the chip is still continuous, but a few pieces of the sawteeth are going to separate with their adjacent ones along the shear surfaces (see Fig. 5e). It is easy to imagine that, as further increasing the cutting speed to an extremely high level,
Table 1 Chemical compositions and heat treatments for Ti-6Al-4V

\begin{tabular}{lllllllll}
\hline \multicolumn{1}{ll}{ Component wt. \% } & \multicolumn{1}{l}{$l$} & \multicolumn{3}{c}{ Heat treatments } \\
\hline $\mathrm{Ti}$ & $\mathrm{Al}$ & $\mathrm{V}$ & $\mathrm{Fe}$ & $\mathrm{N}$ & $\mathrm{H}$ & $\mathrm{O}$ & $\mathrm{C}$ & Annealed at $973 / 2 \mathrm{~h}$, quick cool \\
$\mathrm{Bal}$ & 5.99 & 4.2 & 0.2 & 0.004 & 0.005 & 0.1 & 0.01 & \\
\hline
\end{tabular}


Fig. 1 Diagram of the light-gasgun-based cutting setup

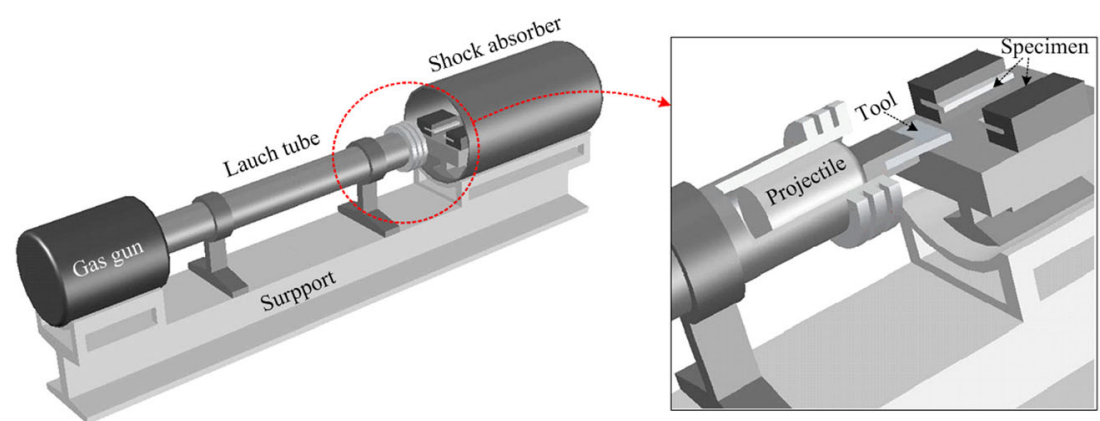

all the sawteeth will separate with each other along the significantly developed shear bands, and the discontinuously segmented chip flow will be imminent.

All of these show clearly that the evolution of the shear band between the sawteeth is the key reason for the emergence of discontinuously segmented chip flow. However, the necessary evolution degree of the shear band for the onset of discontinuous chip flow is unclear, and whether the shear band is fully matured when the segmentation occurs cannot be determined yet. This will be further investigated through the examination of the microstructure of the tool-chip contact surfaces.

The evolution of the microstructure of the tool-chip contact surface with cutting speed (before segmentation) is shown in Fig. 6.

The cutting process is stable at very low cutting speed, and the tool-chip contact surface is quite smooth (see Fig. 6a, d).

For the serrated chip flow, dimpled regions are clearly evident in the tool-chip contact surface( see Fig. 6b, e). The dimples are elongated along the chip flow direction, and the dimple regions are regularly distributed, which are approximately $60 \mu \mathrm{m}$ apart (at $V=7.8 \mathrm{~m} / \mathrm{s}$ ). This is in accordance with the shear band space $L_{c}$ marked in Fig. 3c. This implies that the regularly distributed dimple structure on the tool-chip contact surface is related to the periodical shear banding inside the PSZ. In addition, the periodical dimple features indicate that the chip movement over the tool rake face is stick slip in nature. The dimple feature can be attributed to the welding

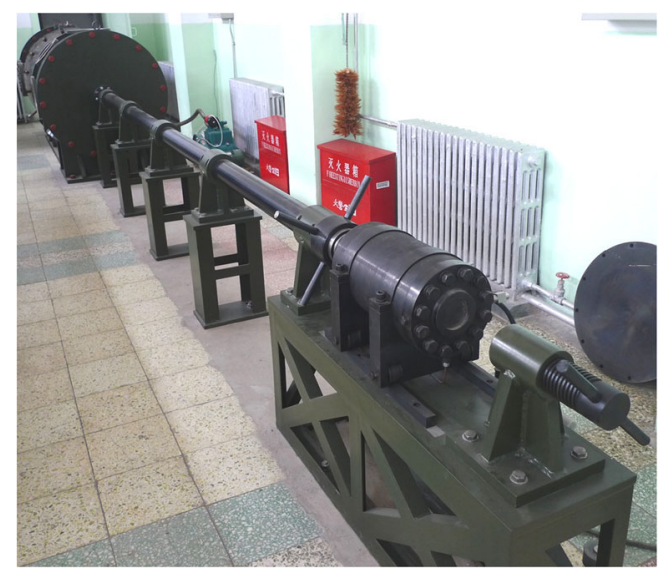

Fig. 2 The actual experimental setup of the chip to the tool face and subsequent fracture upon catastrophic shear localization inside the PSZ [38].

When the cutting speed reaches to $68.9 \mathrm{~m} / \mathrm{s}$, the roots of the shear bands become observable on the tool-chip contact surface (see Fig. 6c). This implies that the shear bands have evolved to a very high level. In this case, local melting occurs along the root of the shear band (see Fig. $6 \mathrm{f}, \mathrm{h}, \mathrm{i}$ ). This is resulted from the high temperature rise caused by the highrate shear localization. It also can be imagined from Fig. 6 that, as further increasing the cutting speed to an extremely high level, the shear band can be fully melted; thus, the chip will be segmented by the fully melted shear bands to render a discontinuous chip flow.

It should be noted that the fully melted shear band has actually lose its bearing capability, which is regarded to be fully matured [39]. Therefore, the emergence of discontinuously segmented chip flow can be attributed to the full development of the shear bands located between the sawteeth.

\subsection{The microstructure evolution after segmentation}

The discontinuously segmented chip formation process is schematically presented in Fig. 7. The chip is segmented by the fully developed shear bands to be pieces of isolated sawteeth. Each sawtooth has four surfaces: free surface, tool-chip contact surface, and two shear fracture surfaces, namely the upper one and the lower one (see Fig. 7). In what follows, the evolutions of the microstructures of the two fracture surfaces and the tool-chip contact surface with cutting speed were investigated.

Figure 8 shows the microstructure of the lower shear fracture surface evolving with cutting speed; the view direction is shown in Fig. 7 (view direction 1).

At $V=86.5 \mathrm{~m} / \mathrm{s}$, the chip flow just changes from continuously serrated to discontinuously segmented. In this case, melt droplets can be wildly observed on the lower shear fracture surface (see Fig. 8a, e, f). Similar to the periodical melting bands observed on the tool-chip interface just before the segmentation occurs (see Fig. 6c, f), the melt droplets are resulted from the high temperature rise caused by the large shear plastic deformation occurring inside the fully matured shear bands. The widely observed melt droplets demonstrate, again, 
Fig. 3 The chip flow patterns at different cutting speeds

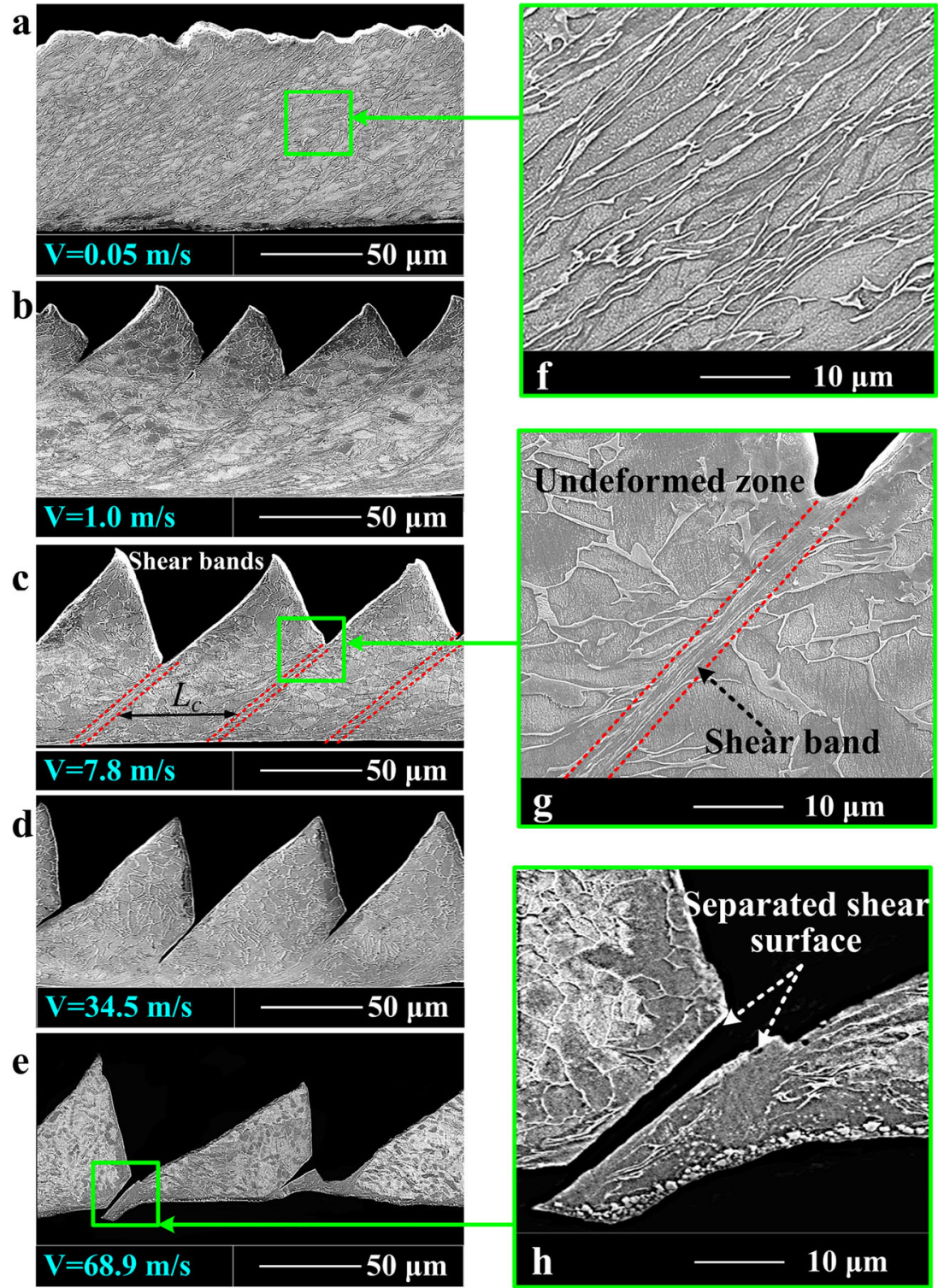

that the onset of discontinuously segmented chip flow is resulted from the full development of the shear bands. The melt droplet, however, disappears as the cutting speed increases to an ultra-high level (see Fig. 8b-d), and the lower shear fracture surface turns out to be smooth at higher cutting speeds.

Moreover, at $V=86.5 \mathrm{~m} / \mathrm{s}$, the elongated dimple structures are clearly visible on the front area of the fracture surface (near the free surface) (see Fig. 8j). This means that the chip segmentation process is dominated by ductile shear fracture. With further increasing the cutting speed to much higher values $(V=128.5,168.4,210.3 \mathrm{~m} / \mathrm{s})$, the dimple decreases while the ductile fracture induced smooth area increases (see Fig. 8k$\mathrm{m})$. This indicates that there exists a ductile-brittle transition of the shear fracture between the sawteeth. According to the research of Fukumasu et al. [40], increasing strain rate reduces the fractural toughness of titanium alloy. This means that the work material could change to be much more brittle at higher cutting speed. So, when the cutting speed reaches a critical

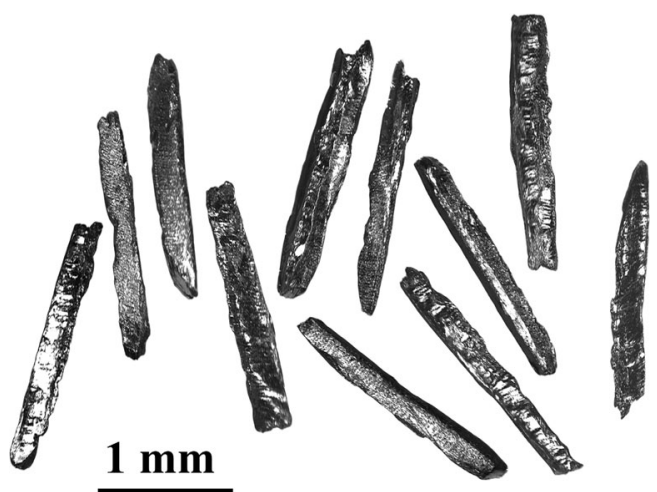

Fig. 4 The discontinuously segmented chips obtained at $V=86.5 \mathrm{~m} / \mathrm{s}$ 




$\mathbf{a}$

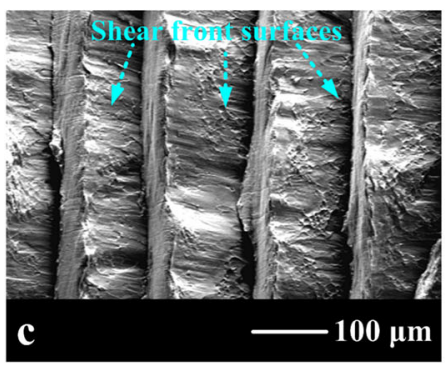

$\mathrm{V}=\mathbf{7 . 8 \mathrm { m } / \mathrm { s }}$

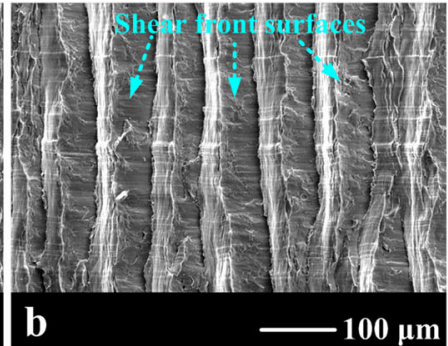

$\mathrm{V}=1.0 \mathrm{~m} / \mathrm{s}$

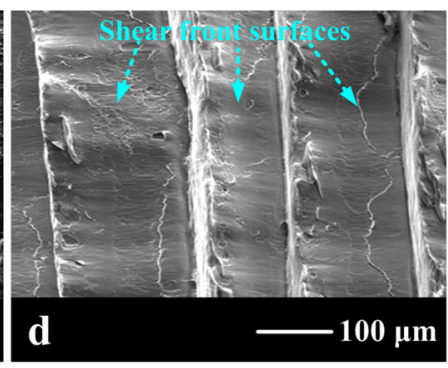

$\mathrm{V}=34.5 \mathrm{~m} / \mathrm{s}$

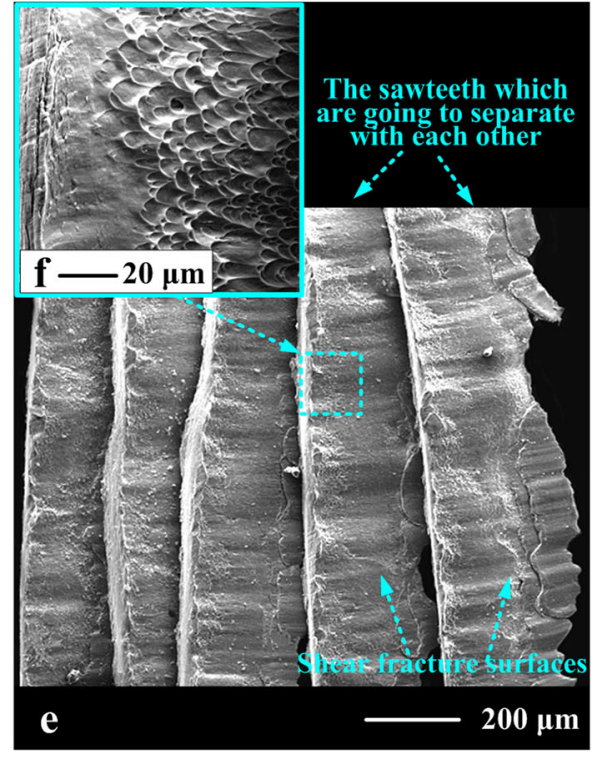

$\mathrm{V}=68.9 \mathrm{~m} / \mathrm{s}$

Fig. 5 The microstructure of the chip free surface at different cutting speeds

high level, the fracture between the sawteeth changes to be dominated by ductile fracture. As thus, the plastic deformation inside the PSZ is restricted, and the heat generation is reduced. As a result, the temperature in the PSZ decreases. This could be the reason why the melt droplet disappears on the shear fracture surface at much higher cutting speeds.
The evolutions of the microstructures of upper shear fracture surface and tool-chip contact surface with cutting speed for discontinuously segmented chip are shown in Fig. 9.

The examination of the upper shear fracture surface also shows a decrease tendency of the dimple with increasing the cutting speed (see Fig. 9k-n). This is in accordance with that

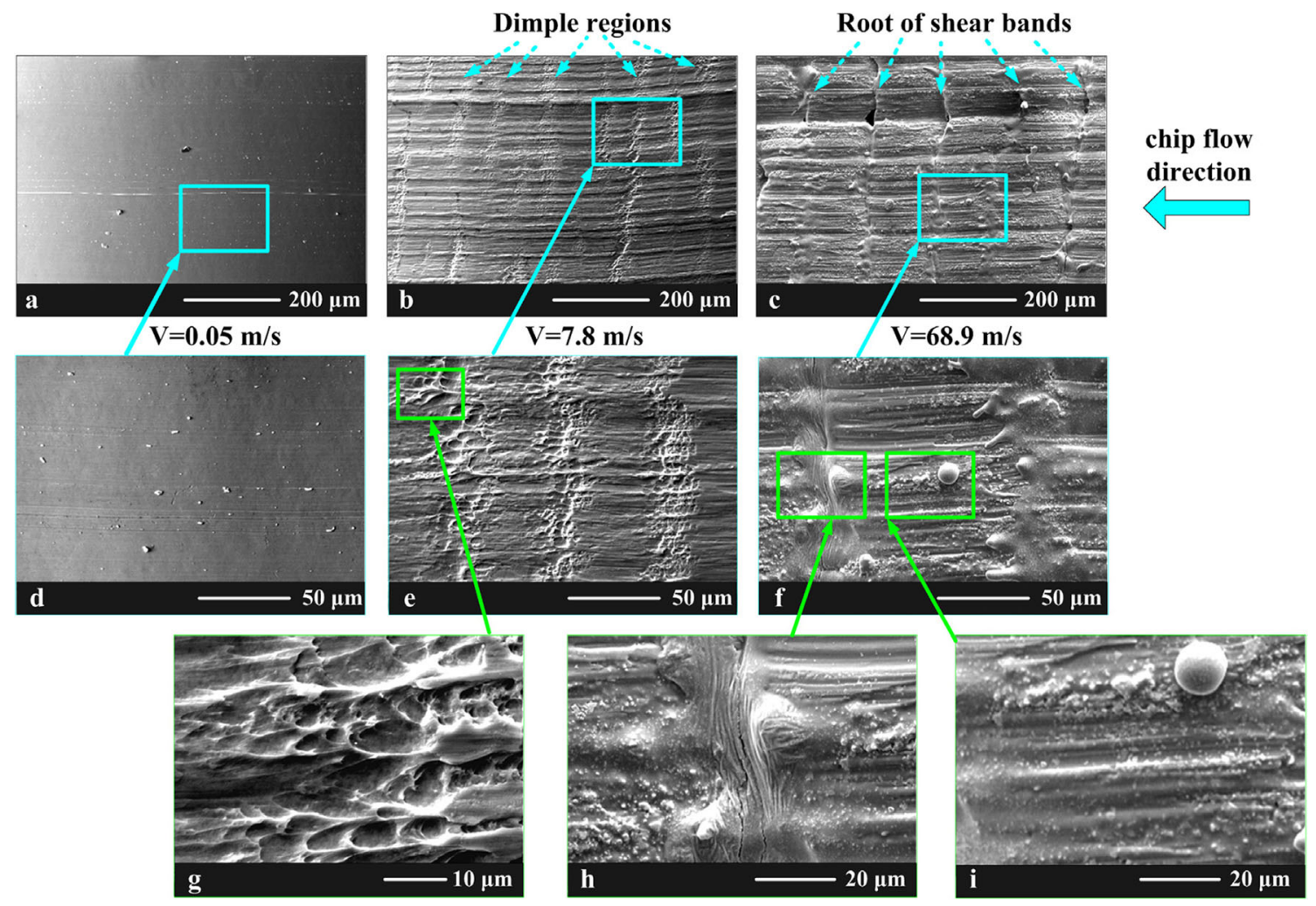

Fig. 6 Tool-chip contact surfaces at different cutting speeds 


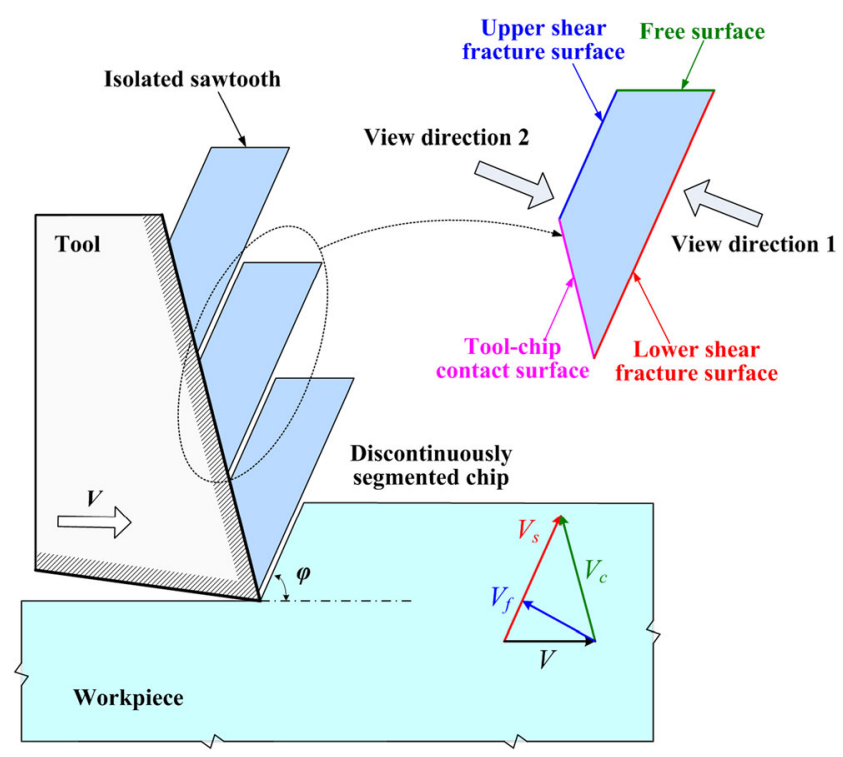

Fig. 7 Schematic for the discontinuously segmented chip formation process

of the lower shear fracture surface, which demonstrates again the existence of the ductile-brittle transition of the dominated shear fracture mechanism.
When the discontinuously segmented chip just forms at $V=86.5 \mathrm{~m} / \mathrm{s}$, the tool-chip contact surface is covered by melt droplets, as shown in Fig. 9a, e, f. The melt droplets are caused by the high temperature rise induced from the severe tool-chip friction. At $V=86.5 \mathrm{~m} / \mathrm{s}$, some of the chip materials on the contact surface melt under the high contact temperature. The melted materials then solidify after they move away from the tool-chip contact zone. Consequently, the melt droplets form on the tool-chip contact surface.

As increasing the cutting speed to a much higher value $(V=128.5 \mathrm{~m} / \mathrm{s})$, the melt droplets disappear, but the flow marks of liquid metal become evident on the contact surface (see Fig. 9b, g, h). At such high cutting speed, the friction between the tool and the chip becomes more severe, and the whole chip materials on the contact surface melt. Then, the flow marks of the melted materials leave on the contact surface after the chip flows away from the tool face.

It can be seen from Fig. 9g, i, $\mathrm{j}$ that the flow marks become more obvious as the cutting speed increases to a higher level. At ultra-high cutting speeds, the chip materials near the contact surface are softened by the high contact temperature. The high-temperature-effected zone becomes deeper at a higher cutting speed, giving rise to a thicker soften layer. It is worth noting that the sticking friction occurs on the tool-chip

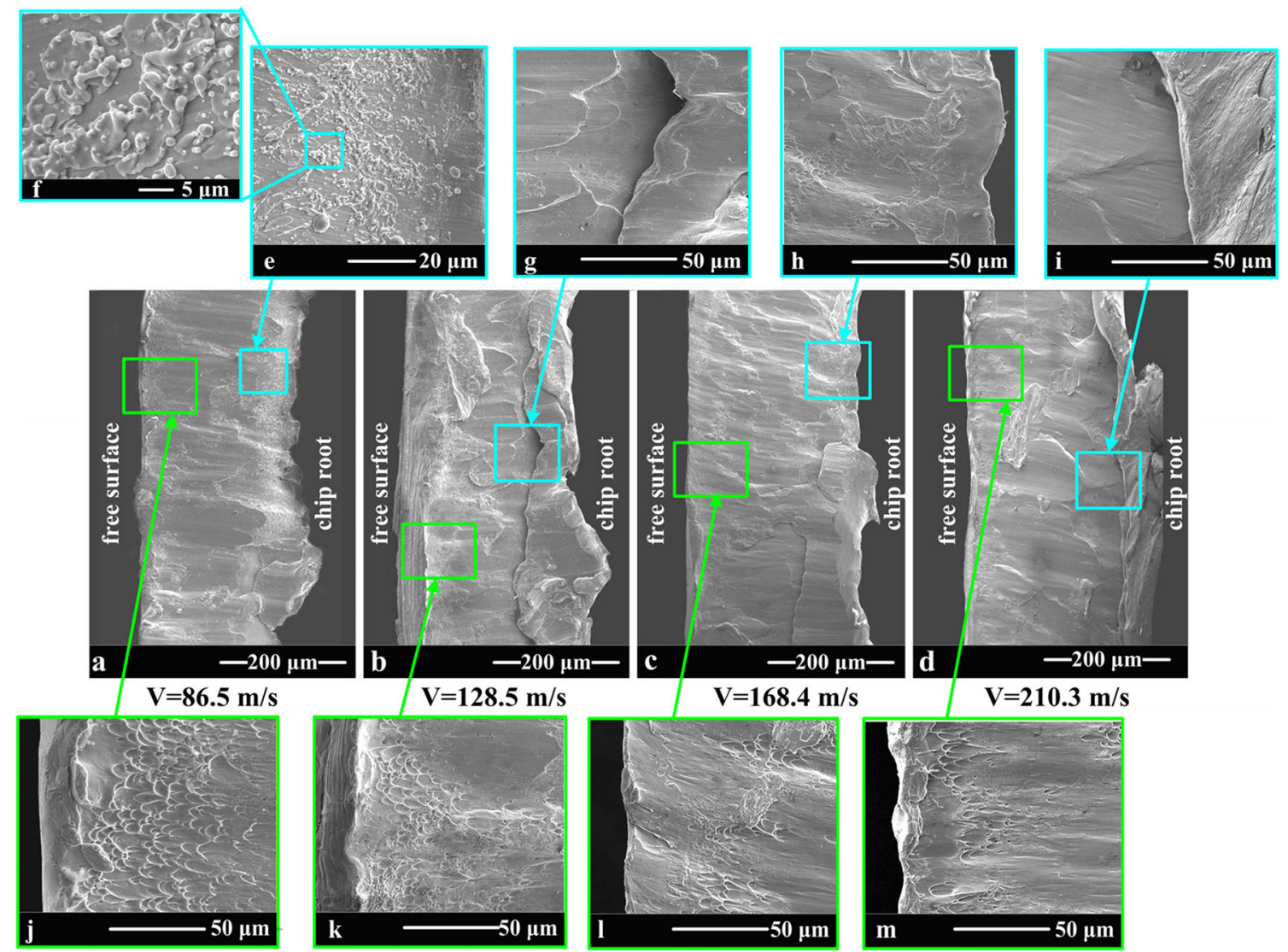

Fig. 8 The microstructures of the lower shear fracture surface at different cutting speeds 


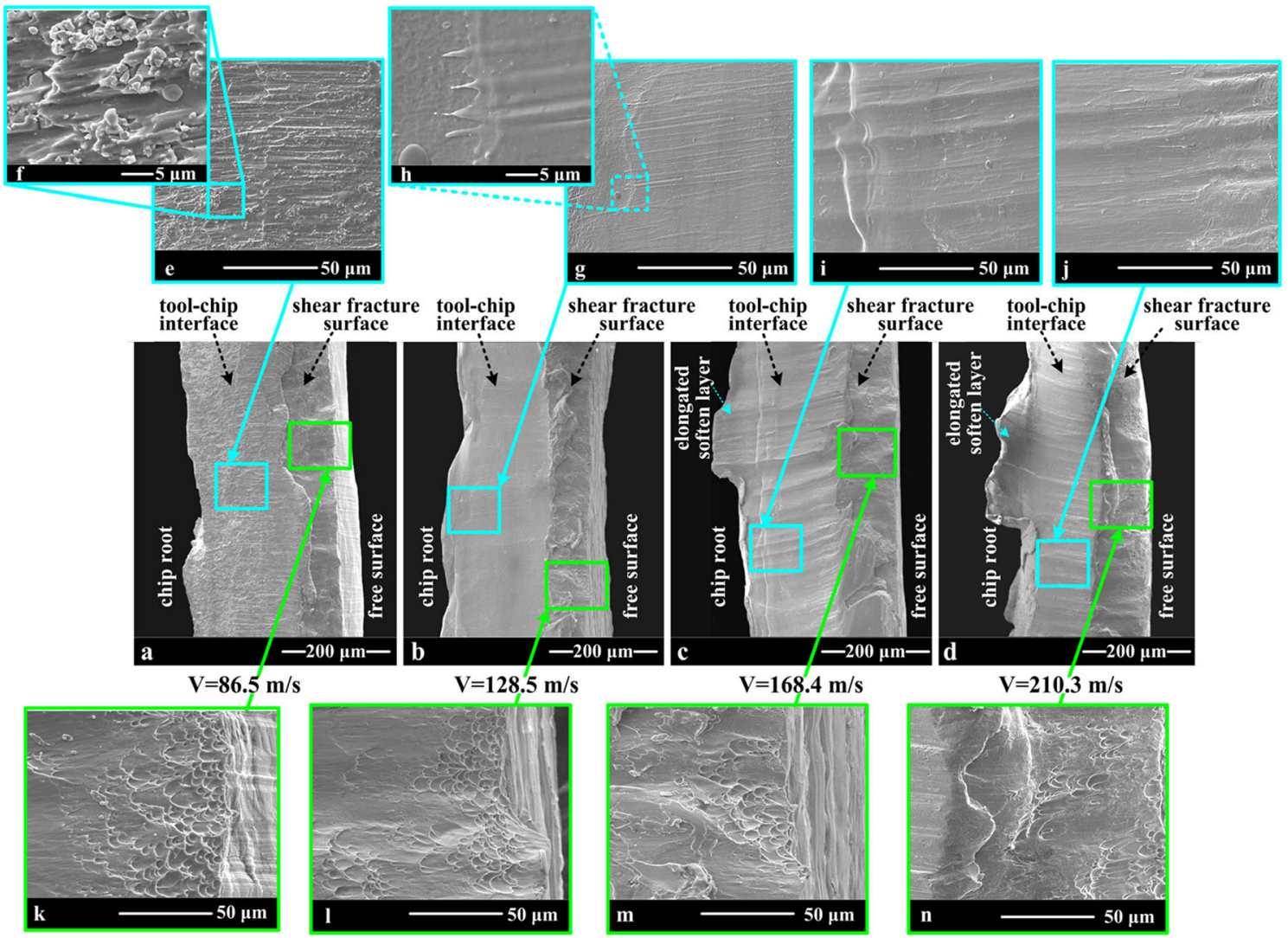

Fig. 9 Microstructures of the upper shear fracture surface and the tool-chip contact surface at different cutting speeds

interface during cutting [41, 42]. Thus, the soften layer is dragged by the severe tool-chip friction during the chip formation process, resulting in an elongated layer at the chip root (see Fig. 9c, d). The elongated soften layer becomes more obvious at higher cutting speeds.

All of these demonstrate that, after the chip is segmented at an extremely high cutting speed, the tool-chip interface temperature is maintained at a high level, and the hightemperature-effected zone becomes deeper with further elevating the cutting speed.

\section{Onset criterion of discontinuously segmented chip flow}

The high-speed cutting experimental results have shown that the transition from continuously serrated chip flow to discontinuously segmented chip flow is dominated by the evolution of shear band. Therefore, the emergence of discontinuously segmented chip flow can be investigated by building a shear band evolution model for the serrated chip flow. In our previous work [43], we have already presented a momentum diffusion-based shear band evolution model to predict the shear band spacing for the serrated chip flow. In what follows, the momentum diffusion-based shear band evolution model was further developed to predict the onset of discontinuously segmented chip flow.

\subsection{Modeling the shear band evolution in serrated chip flow}

Although the full governing equations and various specializations for the shear band evolution model have been described in our previous paper [43], the essentials will be sketched again for clarity.

The formation process of the serrated chip is schematically presented in Fig. 10a. The workpiece is motionless, and the cutting tool moves with a constant cutting speed $V$. Before shear banding, all the deformation of the material occurs within the PSZ which is assumed to be parallel-sided with a finite thickness $h$ [44]. The lower-boundary $C D$ is taken as motionless, and the upper-boundary $E F$ moves with a constant shear velocity $V_{s}$.

When the serrated chip forms, shear banding initiates at the center of the PSZ (along $A B$ ). The shear banding results in stress release within the shear band, which further propagates outward. Consequently, two rigid regions form between the shear band and the plastic deformation zones due to the momentum diffusion caused by the stress relaxation. 




Fig. 10 Theoretical model. a Serrated chip flow model. b The corresponding shear band evolution model

The shear band relaxation process is schematically shown in Fig. 10b. Some necessary assumptions are made as follows:

1. The shear band is assumed to be much thinner than the PSZ [45, 46]. Thus, the evolution of the shear band can be described by the boundary displacement at $y=h / 2$. The boundary displacement $\psi$ increases as the shear band evolves.

2. The two rigid regions caused by the stress relax are assumed to be have a same width $\xi$. Thus, the lower and upper half regions of the PSZ are essentially symmetric.

3. A linear relaxation of the shear stress is used to approximately describe the relation of the unloading stress inside the shear band $[45,46]$,

$\tau(t)=\tau_{y}(1-X(t))$

where $t$ is the evolution time of the shear banding and $\tau_{y}$ is the initial yield shear stress within the plastic region which is treated as constant. The parameter $X(t)$ is defined to describe the evolution degree of the shear band, which is given by

$X(t)=\frac{\psi(t)}{\psi_{c}}(0 \leq X \leq 1)$

where $\psi_{\mathrm{c}}$ is the critical shear displacement at which the shear band stress vanishes and the shear band is regarded to be fully matured. When the evolution degree reaches $X=1$, the shear band is fully developed. In this case, the shear band loses its bearing capacity and the discontinuously segmented chip flow will be imminent.
As shear band evolves, the shear band stress decreases, and the rigid-plastic interface propagates outward. The governing equations controlling this process can be given as follows:

$\frac{d \psi(t)}{d t}=\frac{V_{s}}{h} \xi(t)$

$\rho \gamma_{0} \xi(t) \frac{d \xi(t)}{d t}=\tau_{y}-\tau(t)$

$\tau(t)=\tau_{y}[1-\alpha \theta(t)]+\eta \cdot \frac{2}{a} \frac{d \psi(t)}{d t}+\frac{4 c}{a^{3}} \psi$

$\frac{d \theta(t)}{d t}=\frac{2 \tau(t)}{\rho C_{p} a} \frac{d \psi(t)}{d t}-\frac{2 \lambda}{a^{2}} \theta(t)-\frac{V \sin \varphi}{a} \theta(t)$

where Eq. (3) is the compatible equation for the slip boundary, Eq. (4) is the momentum equation, Eq. (5) is the constitutive equation describing the plastic flow inside the shear band, and Eq. (6) is the energy equation governing the thermo-mechanical deformation of shear band. In these equations, $\rho$ is the mass density, $C_{p}$ is the thermal capacity, $\lambda$ is the thermal diffusivity, $\theta$ is the temperature rise inside shear band, $\alpha$ is the thermal softening coefficient, $\eta$ is the strain rate-hardening coefficient, $c$ is the strain gradient coefficient, $\varphi$ is the shear angle, $\omega$ is the tool rake angle, and $a$ is the shear band thickness.

In Eq. (5), the first term in the right side of the equation reflects the thermal softening effect, the second term reflects the strain rate-hardening effect, and the third term reflects the strain gradient effect. It should be pointed out that, when the shear band forms, the strain gradient is very significant in the shear band. And, according to the researches of Aifantis [47] and Tsagrakis and Aifantis [48], the higher-order strain gradient affects the initiation and propagation of the shear band. So, in this work, we take the strain gradient effect into consideration. 
By using Eqs. (1)-(6) with the initial condition $X(0)=\psi(0)=\xi(0)=0$, an implicit relation of the ultimate dissipation energy with shear band thickness is obtained,

$$
\begin{array}{r}
\frac{2}{X_{u}} \bar{\Gamma}_{u} \bar{a}-\bar{a}^{2}-\frac{(1+M \bar{a})}{X_{u}^{1 / 3}\left(2-X_{u}\right)^{2 / 3}} \bar{\Gamma}_{u}^{2 / 3}-R\left(\frac{\bar{a}_{u}^{1 / 3}}{X_{u}^{2 / 3}\left(2-X_{u}\right)^{1 / 3}}+\frac{4(1+M \bar{a})}{3 X_{u}\left(2-X_{u}\right)} \frac{\bar{\Gamma}_{u}}{\bar{a}}\right) \\
-S \frac{8}{3\left(2-X_{u}\right) X_{u}}\left(\frac{\bar{\Gamma}_{u}}{\bar{a}}+\frac{(1+M \bar{a})}{\left(2-X_{u}\right)^{2 / 3} X_{u}^{1 / 3}} \frac{\Gamma_{u}^{5 / 3}}{\bar{a}}\right)=0
\end{array}
$$

where $X_{u}$ is the ultimate evolution degree of the shear band during chip formation. $\Gamma_{u}$ is the total energy dissipation during the whole shear band relaxation process, which is defined as $\Gamma_{u}=\left(2-X_{u}\right) \tau_{y} \psi_{u}$. The other parameters in Eq. (7) are defined as

$$
\begin{aligned}
& \bar{a}=a\left(\frac{9 \rho^{3} C_{p}^{2} \lambda^{3}}{\tau_{y}^{3} \alpha^{2} \gamma_{0}}\right)^{-1 / 4}, \quad \bar{\Gamma}_{u}=\frac{\Gamma_{u} \alpha}{2 \rho c}\left(\frac{9 \rho^{3} C_{p}^{2} \lambda^{3}}{\tau_{y}^{3} \alpha^{2} \gamma_{0}}\right)^{-1 / 4}, \quad M=\frac{V_{f} a_{0}}{2 \lambda} \\
& R=2 \eta \sqrt{\frac{\gamma_{0}}{\rho \lambda \tau_{y}}}, \quad S=c\left(\frac{\gamma_{0}}{\tau_{y} \rho \lambda^{3}}\right)^{1 / 2}
\end{aligned}
$$

The dimensionless number $M$ reflects the effect of the material convection which is caused by the rapid chip flow, and $R$ and $S$ reflect the effect of rate hardening and strain gradient hardening of material, respectively.
Using the minimum energy principle $\partial \Gamma_{u} / \partial a=0$ $[45,46]$, another relation between the dissipation energy and optimum shear band thickness is obtained:

$$
\begin{array}{r}
\frac{2}{X_{u}} \bar{\Gamma}_{u}-2 \bar{a}-\frac{M}{X_{u}^{1 / 3}\left(2-X_{u}\right)^{2 / 3}} \bar{\Gamma}_{u}^{2 / 3}-R\left(\frac{\bar{\Gamma}_{u}^{1 / 3}}{X_{u}^{2 / 3}\left(2-X_{u}\right)^{1 / 3}}-\frac{4}{3 X_{u}\left(2-X_{u}\right)} \frac{\bar{\Gamma}_{u}}{\bar{a}}\right) \\
+S \frac{8}{3\left(2-X_{u}\right) X_{u}}\left(\frac{\bar{\Gamma}_{u}}{\bar{a}^{2}+\frac{3(1+2 M \bar{a})}{\left(2-X_{u}\right)^{2 / 3} X_{u}^{1 / 3}} \frac{\Gamma_{u}^{5 / 3}}{\bar{a}^{4}}}\right)=0
\end{array}
$$


Solving the system of Eqs. (7) and (9), the ultimate energy dissipation $\Gamma_{u}$ is determined.

\subsection{Onset criterion for the discontinuously segmented chip flow}

When the transition of serrated to discontinuously segmented chip flow occurs, the shear band in the serrated chip is fully developed, namely, $X_{u}=X_{c}=1$. In this case, Eqs. (7) and (9) reduce to

$$
\begin{aligned}
& 2 \bar{\Gamma}_{c} \bar{a}-\bar{a}-(1+M \bar{a}) \bar{\Gamma}_{c}^{2 / 3} \\
& -R\left(\bar{a}_{c}^{1 / 3}+\frac{4(1+\bar{a} M)}{3} \frac{\bar{\Gamma}_{c}}{\bar{a}_{c}}\right) \\
& -\frac{8}{3} S\left(\frac{\bar{\Gamma}_{c}}{\bar{a}}+(1+M \bar{a}) \frac{\Gamma_{c}^{5}}{\bar{a}}\right)=0 \\
& 2 \bar{\Gamma}_{c}-2 \bar{a}-M \bar{\Gamma}_{c}^{2} / 3-R\left(\bar{\Gamma}_{c}^{1 / 3}-\frac{4 M}{3} \frac{\bar{\Gamma}_{c}}{\frac{\bar{a}}{2}}\right) \\
& +S \frac{8}{3}\left(\frac{\bar{\Gamma}_{c}}{\bar{a}^{2}}+(3+2 M \bar{a}) \frac{\Gamma_{c}^{5 / 3}}{\bar{a}^{4}}\right) \\
& \quad=0
\end{aligned}
$$

where $\bar{\Gamma}_{c}=\frac{\Gamma_{c} \alpha}{2 \rho c}\left(\frac{9 \rho^{3} C_{p}^{2} \lambda^{3}}{\tau_{y}^{3} \alpha^{2} \gamma_{0}}\right)^{-1 / 4}$, in which $\Gamma_{c}$ reflects the total energy dissipation for the fully developed shear band. Solving the equation system of Eqs. (10) and (11), the energy dissipation for the fully developed shear band can be determined.

Noting that, during the shear band relaxation process, the tool exerts a constant shear stress $\tau_{y}$ on the upper boundary of the PSZ, causing the upper boundary to move with a constant velocity $V_{s}$ (see Fig. 10b). Therefore, during the whole shear banding process, the energy inputted into the PSZ, or the energy dissipated in the PSZ, can be determined by multiplying the shear stress by its moving distance, that is

$$
W_{\text {input }}=\tau_{y} \cdot V_{s} \cdot t_{c}
$$

where $t_{c}$ is the critical evolution time for the fully matured shear band, which can be determined by solving Eqs. (1)-(6) with setting $X(t)=X_{u}=1$, that is

$t_{c}=\frac{1}{\tau_{y}}\left(\frac{18 \rho \Gamma_{c}^{2}}{\gamma_{0}}\right)^{1 / 3}$

It should be pointed out that the energy inputted into the PSZ is smaller than the total energy input during cutting, because the energy can be dissipated not only in the PSZ but also in the secondary shear zone, where large plastic deformation and friction occur. Here, we are focusing on the energy dissipation in the PSZ. It can be noted that the plastic shear deformation almost takes place inside the shear band for the serrated chip flow, and the material outside the shear band remains almost undeformed, as shown in Fig. 3g. Therefore, we further assume that all the energies inputted into PSZ are dissipated through shear banding.

To make sure that the shear band can be fully developed to result in a discontinuously segmented chip flow, the inputted energy $W_{\text {input }}$ should be larger than the whole dissipation energy for the fully matured shear band $\Gamma_{c}$. As thus, the critical condition for the onset of discontinuously segmented chip flow can be described by

$\Phi=W_{\text {input }} / \Gamma_{c}=V_{s}\left(\frac{18 \rho}{\gamma_{0} \Gamma_{c}}\right)^{1 / 3} \geq 1$

Once the critical condition is achieved, namely $\Phi \geq 1$, the shear band can be fully developed. In this way, the flow stress inside the shear band decreases to zero, and the shear band lose its bearing capacity. Subsequently, all the sawteeth in the serrated chip separate with their adjacent ones along the fully developed shear bands, giving rise to a discontinuously segmented chip flow.

\subsection{Validation for the onset criterion}

For given cutting conditions and work material, the energy dissipation of the fully matured shear band $\left(\Gamma_{c}\right)$ can be determined by solving Eqs. (10) and (11). Thus, the onset of discontinuously segmented chip flow can be predicted according to the criterion (14). In this section, the predicted results will be compared with the experimental findings for Ti-6Al-4V to validate the onset criterion.

It should be noted that, before shear banding, the work material has undergone a homogeneous plastic shear 
Table 2 Mechanical properties and parameters for Ti-6Al-4V

\begin{tabular}{lll}
\hline Properties and parameters & Notation & Value \\
\hline Density & $\rho$ & $4430 \mathrm{~kg} \mathrm{~m}^{-3}$ \\
Thermal diffusivity & $\lambda$ & $2.95 \times 10^{-6} \mathrm{~m}^{2} \mathrm{~s}^{-1}$ \\
Specific heat & $c$ & $520 \mathrm{~J} \mathrm{~kg}^{-1} \mathrm{~K}^{-1}$ \\
Initial yield stress for J-C model & $\tau_{A}$ & $452 \mathrm{MPa}$ \\
Hardening modulus for J-C model & $\tau_{B}$ & $287 \mathrm{MPa}$ \\
Strain rate dependency coefficient for J-C model & $C$ & 0.028 \\
Work-hardening exponent for J-C model & $n$ & 0.28 \\
The reference stain rate for J-C model & & $1.73 \times 10^{-5} \mathrm{~s}^{-1}$ \\
Thermal softening coefficient & $\alpha$ & $6.5 \times 10^{-4} \mathrm{~K}^{-1}$ \\
Strain rate-hardening coefficient & $\eta$ & $0.75 \mathrm{~Pa} \mathrm{~s}^{-1}$ \\
Strain gradient coefficient & $c$ & $2.5 \times 10^{-5} \mathrm{~N}$ \\
\hline
\end{tabular}

deformation at constant shear rate $\gamma_{0}$. So, the initial yield stress $\tau_{y}$ is related to the homogeneous shear strain $\gamma_{0}$ and the shear strain rate $\gamma_{0}$, namely, $\tau_{y}=\tau_{y}\left(\gamma_{0}, \gamma_{0}\right)$. Following the Johnson-Cook (J-C) model, the initial yield stress is given in the form of

$\tau_{y}\left(\gamma_{0}, \gamma_{0}\right)=\left(\tau_{A}+\tau_{B}\left(\frac{\gamma_{0}}{\sqrt{3}}\right)^{n}\right)\left[1+C \ln \left(\frac{\gamma_{0}}{\gamma_{\text {ref }}}\right)\right]$

where $\tau_{A}, \tau_{B}, n, C$, and $\gamma_{\text {ref }}$ are the parameters for J-C model. They are defined in Table 2 together with other mechanical parameters for Ti-6Al-4V [43, 49].

The shear velocity and the average shear strain for the homogeneous deformation can be estimated by $V_{s}=V \cos \omega /$ $\cos (\varphi-\omega)$ and $\gamma_{0}=\cos \omega / 2 \sin \varphi \cos (\varphi-\omega)$, respectively [44]. The shear angle $\varphi$ is set to be $45^{\circ}$ according to the experimental findings, and the PSZ thickness $h$ is set to be $1 / 10$ of the uncut chip thickness [21].

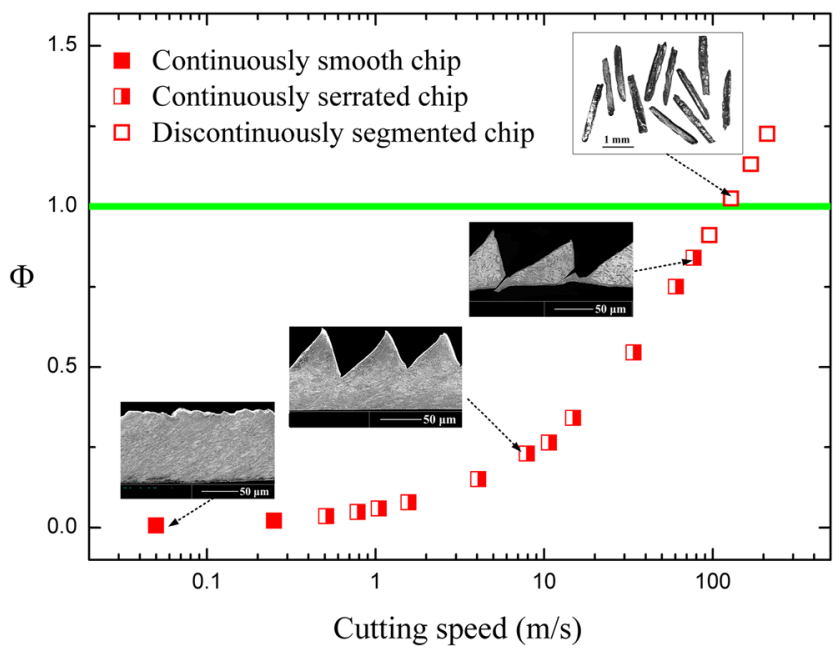

Fig. $11 \Phi$ evolves with cutting speed for Ti-6AL-4V $(b=100 \mu \mathrm{m}$, $\omega=0^{\circ}$ )
Solving Eqs. (10) and (11) with the cutting conditions and the mechanical parameters listed in Table 2, the energy dissipation $\Gamma_{c}$ is determined. In this way, the ratio of the input energy to the total dissipation energy in the PSZ is achieved according to Eq. (14); thus, the onset of segmented chip flow can be investigated.

The evolution of the ratio of input energy to the total dissipation energy $(\Phi)$ with cutting speed for Ti-6Al-4V is shown in Fig. 11. It can be seen that $\Phi$ increases with increasing the cutting speed, which means that increasing the cutting speed promotes the onset of discontinuously segmented chip flow. This is in accordance with the experimental findings. It also can be seen that the flow patterns are discontinuously segmented in the zone of $\Phi>1$ and that the continuously smooth and serrated flow patterns fall in the zone of $\Phi<1$. Most importantly, the transition of the flow pattern from continuously serrated to discontinuously segmented almost always occurs around $\Phi=1$. This figure implies that the onset criterion

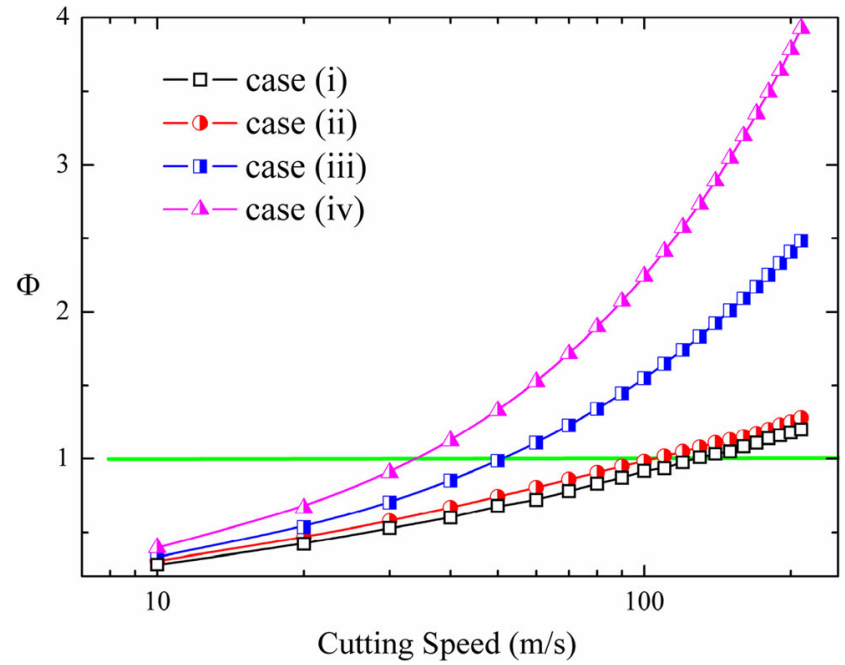

Fig. $12 \Phi$ evolves with cutting speed for Ti-6AL-4V 
(14) allows the prediction of the emergence of discontinuously segmented chip flow.

Furthermore, the influences of the rate hardening and the material convection on the onset of segmented chip flow are further investigated. Four typical cases are discussed: case (i), in which the effects of strain gradient hardening, rate hardening, and material convection are considered; case (ii), where the effect of strain gradient effect is neglected, only the rate hardening and material convection are considered; case (iii) in which the material convection and strain gradient hardening are neglected, only the rate-hardening effect of the work material is considered; and case (iv), in which the effects of strain gradient hardening, rate hardening, and material convection are neglected. For case (iv), the dimensionless numbers $M$, $R$, and $S$ are vanished in Eqs. (10) and (11). For case (iii), $M$ and $S$ vanish. For case (ii), only $S$ vanishes.

$\Phi$ evolving with cutting speed for these three cases is presented in Fig. 12. Comparing cases (ii), (iii), and (iv) with case (i), it can be seen that neglecting the effects of material convection, rate hardening, and strain gradient hardening leads to a lower cutting speed at which the shear band is fully developed $(\Phi=1)$. This figure implies that the material convection, rate hardening, and strain gradient hardening limit the tendency toward segmentation. It should be noted that the material convection, rate hardening, and strain gradient hardening hinder the thermal softening and, hence, the shear relaxation [43]. This leads to a larger energy dissipation for the shear band evolution and thus retards the emergence of discontinuously segmented chip flow.

\section{Conclusions}

In conclusion, orthogonal cutting experiments were performed on Ti-6Al-4V over a very wide range of cutting speeds, from 0.05 to $210 \mathrm{~m} / \mathrm{s}$. The transitions of chip flow pattern from continuously smooth to serrated and further to discontinuously segmented with increasing cutting speed were observed. Examination of the evolutions of the microstructures shows clearly that the serrated chip flow can be attributed to the repeated catastrophic shear banding occurred in the PSZ. When the shear band is fully matured, shear fracture takes place near the shear band root, and the discontinuously segmented chip flow emerges. We further investigated the evolution of the shear fracture surfaces of the segmented chips. It reveals that there exists a ductile-brittle transition of the dominated fracture mechanism with increasing the cutting speed to an ultra-high level. More importantly, we developed the momentum diffusion-based shear band evolution model to predict the onset of discontinuously segmented chip flow. It is found that once the energy inputted into the PSZ is large enough for the shear band to fully develop, the discontinuously segmented chip flow will be imminent. We compared the onset criterion with the experimental results, and good agreements were achieved, implying that the onset criterion allows the prediction of the emergence of discontinuously segmented chip flow. At last, the influences of the rate hardening and the material convection on the onset of discontinuously segmented chip flow are further investigated, which shows that both the material convection and the rate-hardening limit the tendency toward segmentation.

Acknowledgments This work was supported by the Nature Science Foundation of China (Grants Nos: 11132011, 11402278), the National Basic Research Program of China (Grant No: 2012CB937500), and the Fundamental Research Funds for the Central Universities (15CX02110A)

\section{References}

1. Salomon CJ (1931) Process for the machining of metals or similarly acting materials when being worked by cutting tools. German Patent, Number 523594, 1931-04

2. Xu D, Feng P, Li W, Ma Y, Liu B (2014) Research on chip formation parameters of aluminum alloy 6061-T6 based on high-speed orthogonal cutting model. Int J Adv Manuf Technol 72:955-962

3. Ma J, Wang F, Jia Z, Xu Q, Yang Y (2014) Study of machining parameter optimization in high speed milling of Inconel 718 curved surface based on cutting force. Int J Adv Manuf Technol 75:269 277

4. Barry J, Byrne G (2002) The mechanisms of chip formation in machining hardened steels. J Manuf Sci Eng 124:528-535

5. Jiang MQ, Dai LH (2009) Formation mechanism of lamellar chips during machining of bulk metallic glass. Acta Mater 57:2730-2738

6. Shaw MC, Vyas A (1993) Chip formation in the machining of hardened steel. CIRP Ann Manuf Technol 42:29-33

7. Pu CL, Zhu G, Yang S, Yue EB, Subramanian SV (2015) Effect of microstructure softening events on the chip morphology of AISI 1045 steel during high speed machining. Int J Adv Manuf Tech

8. Kountanya R, Al-Zkeri I, Altan T (2009) Effect of tool edge geometry and cutting conditions on experimental and simulated chip morphology in orthogonal hard turning of $100 \mathrm{Cr} 6$ steel. J Mater Process Technol 209:5068-5076

9. Wang H, To S, Chan CY, Cheung CF, Lee WB (2010) A study of regularly spaced shear bands and morphology of serrated chip formation in microcutting process. Scr Mater 63:227-230

10. Wang H, To S, Chan CY, Cheung CF, Lee WB (2010) Elastic strain induced shear bands in the microcutting process. Int $\mathrm{J}$ Mach Tool Manuf 50:9-18

11. Wang H, To S, Chan CY, Cheung CF, Lee WB (2011) Dynamic modelling of shear band formation and tool-tip vibration in ultraprecision diamond turning. Int J Mach Tool Manuf 51:512-519

12. Molinari A, Musqua C, Sutter G (2002) Adiabatic shear banding in high speed machining of Ti-6Al-4V: experiments and modeling. Int J Plast 18:443-459

13. Duan CZ, Zhang LC (2012) Adiabatic shear banding in AISI 1045 steel during high speed machining: mechanisms of microstructural evolution. Mater Sci Eng A 532:111-119

14. Cai SL, Dai LH (2014) Suppression of repeated adiabatic shear banding by dynamic large strain extrusion machining. J Mech Phys Solids 73:84-102

15. Wan L, Wang D, Gao Y (2015) The investigation of mechanism of serrated chip formation under different cutting speeds. Int $\mathrm{J}$ Adv Manuf Tech 
16. Recht RF (1964) Catastrophic thermoplastic shear. J Appl Mech 189-193

17. Hou ZB, Komanduri R (1997) Modeling of thermomechanical shear instability in machining. Int J Mech Sci 39:1279-1314

18. Molinari A, Dudzinski D (1992) Stationary shear band in highspeed machining. CR Acad Sci Paris 315:399-405

19. Huang J, Aifantis EC (1997) A note on the problem of shear localization during chip formation in orthogonal machining. J Mater Eng Perform 6:25-26

20. Huang J, Kalaitzidou K, Sutherland JW, Aifantis EC (2007) Validation of a predictive model for adiabatic shear band formation in chips produced via orthogonal machining. J Mech Behav Mater 18:243-263

21. Ye GG, Chen Y, Xue SF, Dai LH (2014) Critical cutting speed for onset of serrated chip flow in high speed machining. Int J Mach Tool Manuf 86:18-33

22. Duan C, Zhang L (2012) A reliable method for predicting serrated chip formation in high-speed cutting: analysis and experimental verification. Int J Adv Manuf Technol 64:1587-1597

23. Gao C, Zhang L (2013) Effect of cutting conditions on the serrated chip formation in high-speed cutting. Mach Sci Technol 17:26-40

24. Sowerby R, Chandrasekaran N (1989) A proposal for the onset of chip segmentation in machining. Mater Sci Eng A 119:219-229

25. Marusich T, Ortiz M (1995) Modelling and simulation of highspeed machining. Int J Numer Methods Eng 38:3678-3694

26. Lin ZC, Lo SP (2001) 2-D discontinuous chip cutting model by using strain energy density theory and elastic-plastic finite element method. Int J Mech Sci 43:381-398

27. Lin ZC, Lin YY (2001) Three-dimensional elastic-plastic finite analysis for orthogonal cutting with discontinuous chip of 6-4 brass. Theor Appl Fract Mech 35:137-153

28. Guo YB, Yen DW (2004) A FEM study on mechanisms of discontinuous chip formation in hard machining. J Mater Process Technol 155-156:1350-1356

29. Liu Z, Su G (2012) Characteristics of chip evolution with elevating cutting speed from low to very high. Int J Mach Tool Manuf 54-55: $82-85$

30. Sutter G, List G (2013) Very high speed cutting of Ti-6Al-4V titanium alloy - change in morphology and mechanism of chip formation. Int J Mach Tool Manuf 66:37-43

31. Gu L, Wang M, Duan C (2013) On adiabatic shear localized fracture during serrated chip evolution in high speed machining of hardened AISI 1045 steel. Int J Mech Sci 75:288-298

32. Cui X, Zhao B, Jiao F, Zheng J (2015) Chip formation and its effects on cutting force, tool temperature, tool stress, and cutting edge wear in high- and ultra-high-speed milling. Int J Adv Manuf Tech
33. Ye GG, Xue SF, Ma W, Jiang MQ, Ling Z, Tong XH, Dai LH (2012) Cutting AISI 1045 steel at very high speeds. Int J Mach Tool Manuf 56:1-9

34. Li A, Zhao J, Zhou Y, Chen X, Wang D (2011) Experimental investigation on chip morphologies in high-speed dry milling of titanium alloy Ti-6Al-4V. Int J Adv Manuf Technol 62:933-942

35. Zhu Z, Sun J, Li J, Huang P (2015) Investigation on the influence of tool wear upon chip morphology in end milling titanium alloy Ti6Al4V. Int J Adv Manuf Tech

36. Wang B, Liu Z (2014) Investigations on the chip formation mechanism and shear localization sensitivity of high-speed machining Ti6Al4V. Int J Adv Manuf Technol 75:1065-1076

37. Cho KM, Chi YC, Duffy J (1990) Microscopic observations of adiabatic shear bands in three different steels. Metall Trans A 21: 1161-1175

38. Barry J, Byrne G, Lennon G (2001) Observations on chip formation and acoustic emission in machining Ti-6Al-4V alloy. Int J Mach Tool Manuf 41:1055-1070

39. Grady DE (1994) Dissipation in adiabatic shear bands. Mech Mater 17:289-293

40. Fukumasu H, Kobayashi T, Toda H, Shibue K (2000) Dynamic fracture toughness of a Ti-45Al-1.6Mn alloy at high temperature. Metall Mater Trans A 31A:2000-3053

41. Ozel T, Altan T (2000) Determination of workpiece flow stress and friction at the chip-tool contact for high-speed cutting. Int J Mach Tool Manuf 40:133-152

42. Sartkulvanich P, Altan T, Göcmen A (2005) Effects of flow stress and friction models in finite element simulation of orthogonal cutting - a sensitivity analysis. Mach Sci Technol 9:1-26

43. Ye GG, Xue SF, Jiang MQ, Tong XH, Dai LH (2013) Modeling periodic adiabatic shear band evolution during high speed machining Ti-6Al-4V alloy. Int J Plast 40:39-55

44. Oxley PLB (1989) Mechanics of machining. Wiley, New York

45. Grady DE, Kipp ME (1987) The growth of unstable thermoplastic shear with application to steady-wave shock compression in solids. J Mech Phys Solids 35:95-118

46. Grady DE (1992) Properties of an adiabatic shear-band process zone. J Mech Phys Solids 40:1197-1215

47. Aifantis EC (1987) The physics of plastic deformation. Int J Plast 3: 211-247

48. Tsagrakis I, Aifantis EC (2015) On the effect of strain gradient on adiabatic shear banding. Metall Mater Trans A 46:4459-4467

49. Lee WS, Lin CF (1998) High-temperature deformation behaviour of Ti6Al4V alloy evaluated by high strain-rate compression tests. J Mater Process Technol 75:127-136 